\title{
Gravidez Abdominal a Termo com Feto Vivo: Relato de Caso
}

\author{
Abdominal Pregnancy at Term with Live Fetus: \\ A Case Report
}

Luiz Carlos Santos, Melania Maria Ramos de Amorim, Edmar Miranda, Gisela de Oliveira Saunders, Elizabete de Oliveira, Jonyplácido Gattás

\section{RESUMO}

Gestação abdominal a termo com feto vivo representa uma raridade obstétrica que cursa com elevada morbimortalidade materna e perinatal. Neste estudo, os autores apresentam um caso de gestação abdominal em uma paciente de 43 anos, em quem o diagnóstico só foi realizado a termo (com 37 semanas), pelos achados clínicos e ecográficos. Realizou-se laparotomia exploradora com extração de recém-nascido vivo do sexo feminino, pesando 2.570 gramas. Os escores de Apgar foram de 3, 6 e 8 no $1^{\circ}, 5^{\circ}$ e $10^{\circ}$ minutos, respectivamente. A placenta se encontrava inserida no omento e foi removida sem complicações. A evolução operatória foi satisfatória e tanto a mãe como a criança obtiveram alta em boas condições.

PALAVRAS-CHAVE: Gravidez ectópica. Gravidez abdominal. Complicações da gravidez. Crescimento fetal retardado.

\section{Introdução}

A gestação abdominal constitui um evento extremamente raro em Obstetrícia; sua incidência varia de 1 para 10.000 a 1 para 64.000 nascimentos ${ }^{1,2}$, sendo mais freqüente nos países subdesenvolvidos ou em desenvolvimento ${ }^{3,4,5}$. Em 1987, Atrash et al. ${ }^{6}$ descreveram uma incidência de 10,9 por 100.000 nascidos vivos nos Estados Unidos, equivalente a 9,2 para 1.000 gestações ectópicas.

$\mathrm{Na}$ maioria desses casos verifica-se o decesso fetal, em decorrência da insuficiência placentária, por conta da má implantação do trofoblasto $^{2,5}$. São ainda comuns as deformidades de membros e face do concepto, bem como a hipoplasia pulmonar, conseqüentes à compressão pelas estruturas abdominais ${ }^{2,7}$. A morbimortalidade materna é elevada ${ }^{1,5,6,8}$, sendo freqüentes complicações cataclísmicas, como a hemorragia do leito placentário ${ }^{2}$. Mais freqüente, todavia, é a

Instituto Materno-Infantil de Pernambuco (IMIP) - Centro de Atenção à Mulher (CAM)

Correspondência:

Melania Maria Ramos de Amorim

IMIP - Coordenação de Ensino

Rua dos Coelhos, 300 - Boa Vista

50070-550 - Recife - PE

e-mail:melania@interway.com.br dificuldade de extração placentária, por conta de sua implantação em vísceras nobres, como o figado, não sendo incomum a decisão do tocólogo de deixar a placenta in situ, no intuito de evitar o sangramento profuso e a lesão das estruturas abdominais ${ }^{2,9}$.

A gestação abdominal a termo com feto vivo apresenta outrossim maior raridade, sendo descritos apenas casos esporádicos na literatura. A sobrevivência neonatal é de cerca de $20 \%$ nas maiores séries publicadas ${ }^{1,2,8}$. Em nosso meio, a maior experiência com gestação abdominal foi descrita por Costa et al. (Recife, PE) $)^{10,11,12}$, porém em poucas ocasiões encontrou-se concepto vivo com condições de sobrevivência neonatal ${ }^{10,12}$. Em 1992, Alencar et al. ${ }^{13}$ descreveram um caso de gestação abdominal avançada com recém-nascido vivo, na Maternidade-Escola Assis Chateuabriand, em Fortaleza. Mendonça et al. ${ }^{14}$ (João Pessoa, PB) também relataram, em 1993, um caso de rotura de gravidez abdominal avançada com feto vivo, que cursou com grave hemorragia. Devido à extrema singularidade, recomenda-se portanto a divulgação de todos os casos que venham a ser diagnosticados.

No presente estudo, os autores relatam sua experiência com um caso de gestação abdominal tardia conduzido no Centro de Atenção à Mulher do IMIP, obtendo-se concepto vivo e evolução 
satisfatória, com sobrevivência materna e neonatal.

\section{Relato do Caso}

S.B.S., 43 anos, gesta IV para I (1 cesárea), procedente de Campestre (interior do estado de Pernambuco), admitida no IMIP em 8/5/1999, com história de dor abdominal desde o início da gestação, que piorava com os movimentos fetais e que tinha se intensificado nos três últimos dias. Negava distúrbios do ritmo intestinal. Desconhecia a data da última menstruação, trazendo contudo ultra-sonografia realizada em sua cidade em 2/2/1999, que evidenciava gestação tópica e que, corrigida, resultava em idade gestacional de 37 semanas. Referia 3 consultas durante o pré-natal, sem intercorrências.

Ao exame físico, encontrava-se em mau estado geral, hipocorada $(++/ 4+)$, desnutrida, desidratada, afebril, anictérica, acianótica, sem edema. A pressão arterial era de $100 \times 60 \mathrm{mmHg}$, a freqüência cardiaca de $100 \mathrm{bpm}$ e a ausculta cardiopulmonar sem anormalidades. O abdome era doloroso à palpação superficial e profunda, verificando-se superficialidade das partes fetais, com altura abdominal de $26 \mathrm{~cm}$. O feto estava em situação longitudinal, não sendo possivel determinar a apresentação. A freqüência cardíaca fetal (FCF) era de $140 \mathrm{bpm}$, auscultada no hipocôndrio esquerdo. Ao toque, colo longo, posterior, impérvio.

As hipóteses diagnósticas aventadas no internamento foram de gestação ectópica avançada com feto vivo, anemia e desnutrição. Foram solicitados hemograma, classificação sangüinea e ultra-sonografia. Realizou-se no mesmo dia o exame ecográfico, com o seguinte laudo: "Gestação única com feto em apresentação pélvica, visibilizado dentro da cavidade abdominal, com boa vitalidade. Placenta inserida na parede anterior da cavidade peritoneal, grau III. Oligoâmnio discreto. Diâmetro biparietal (DBP) de $8,3 \mathrm{~cm}$, comprimento do fêmur $(\mathrm{CF})$ de $6,6 \mathrm{~cm}$ e circunferência abdominal (CA) de $28,1 \mathrm{~cm}$, resultando em idade gestacional de 33 semanas, mais ou menos 1 semana. Útero visibilizado em sua topografia habitual, aumentado de volume, de textura homogênea, sem conteúdo em sua cavidade. Medidas uterinas de 11,7 x 8,5 x 7,7 cm. Ausência de líquido livre em cavidade peritoneal. Conclusão: gestação abdominal com feto vivo no curso da $33^{a}$ semana de gestação, devendo-se aventar a possibilidade de crescimento fetal retardado (CFR), uma vez que a idade gestacional pela ultra-sonografia anterior corrigida é de 37 semanas."

Levando em consideração a idade gestacional de 36 semanas pela ultra-sonografia mais precoce, a suspeita de CFR e a diminuição de líquido amniótico, indicou-se laparotomia exploradora, precedida porém de cuidados préoperatórios. Administrou-se corticoterapia com hidrocortisona $1 \mathrm{~g}$ ev a cada 6 horas por 24 horas, devido à idade gestacional ecográfica de 33 semanas, com a finalidade de acelerar a maturidade pulmonar fetal, previamente à laparotomia. A paciente ficou em jejum, sob infusão de líquidos (Ringer lactato e soro glicosado hipertônico) e recebeu ainda hemotransfusão com 2 (dois) concentrados de hemácias em 9/5/1999, uma vez que apresentava valores de hemoglobina (Hb) de 7,8 g\% e hematócrito (Ht) de 23,4\%.

A hemoglobina pós-transfusão foi de $10 \mathrm{~g} \%$, hematócrito de $29 \%$. Ao mesmo tempo, fez-se a reserva de mais 4 (quatro) concentrados de hemácias. Repetiu-se a ultra-sonografia neste mesmo dia, mantendo-se os achados já descritos no exame inicial e verificando-se boa vitalidade fetal. Manteve-se durante todo o período préoperatório vigilância constante da vitalidade fetal e das condições hemodinâmicas maternas.

Realizou-se laparotomia exploradora em 10/ 5/1999, sob anestesia geral endovenosa (com propofol-Diprivan $\AA$ e Rapifen ${ }^{\circledR}$ ) e inalatória (com Etrane $\left.{ }^{\circledR}\right)$, associada a peridural contínua com neocaína e morfina. A incisão abdominal foi mediana, xifopubiana, encontrando-se a parede abdominal muito adelgaçada, não se evidenciando os músculos retos abdominais. Abrindo-se a cavidade abdominal, encontrou-se imediatamente volumosa tumoração ocupando o abdome inferior e superior, que correspondia ao saco gestacional recoberto pelo omento. Esse saco membranoso encontrava-se roto em sua porção inferior, evidenciando-se exteriorização de um dos pés do concepto (Figura 1).

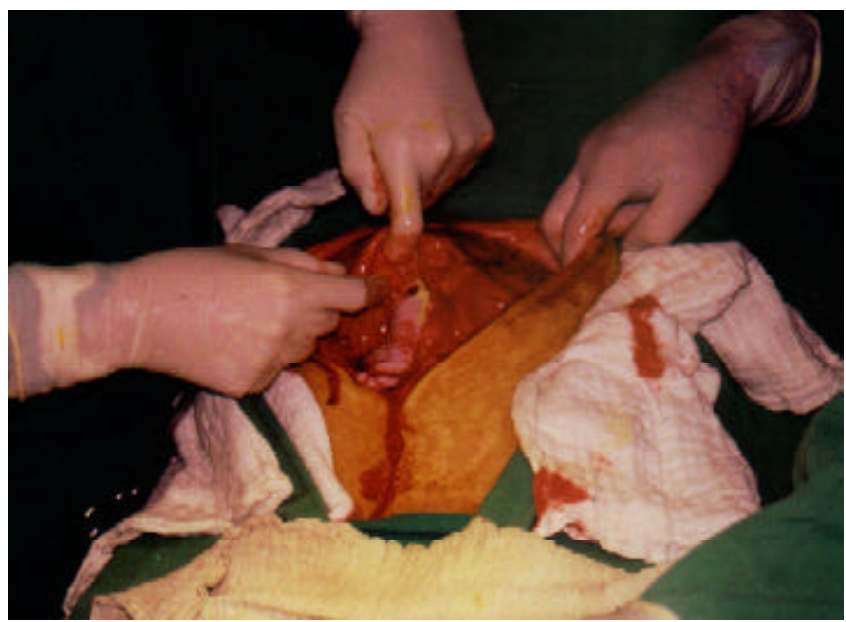

Figura 1 - Abertura do saco constituído pela membrana amniótica recoberta pelo omento visualizando-se o concepto em seu interior. 
Procedeu-se então à abertura do omento e da membrana amniótica, retirando-se recémnascido vivo do sexo feminino, que se encontrava disposto longitudinalmente, com o pólo pélvico na região do abdome inferior. Observou-se líquido amniótico em escassa quantidade, claro, com grumos. Clampeando-se o cordão umbilical, identificou-se a seguir a placenta, bastante volumosa, que se encontrava aderida superiormente ao grande omento e, inferiormente, ao cécum, cólon ascendente e fundo uterino. Todas as aderências foram liberadas, com pinçamento, ressecção e ligadura do epiplon, sem hemorragia importante, retirando-se ao final a placenta completa (Figura 2). Revisou-se a seguir a cavidade abdominal, encontrando-se discreta lesão da serosa do cécum (suturada) e do apêndice, que se encontrava aderido à massa placentária, bem como da trompa esquerda, cujo infundíbulo fora amputado durante a lise de aderências. A conduta adotada foi apendicectomia e salpingectomia esquerda. Realizou-se também ligadura tubária à direita. Efetuou-se lavagem cuidadosa da cavidade abdominal com soro fisiológico e a seguir sutura em bloco da parede abdominal, com fio prolene $\mathrm{n}^{\circ}$ 2 , em pontos separados. A pele foi aproximada com pontos à Donati utilizando-se fio mononylon 3-0.

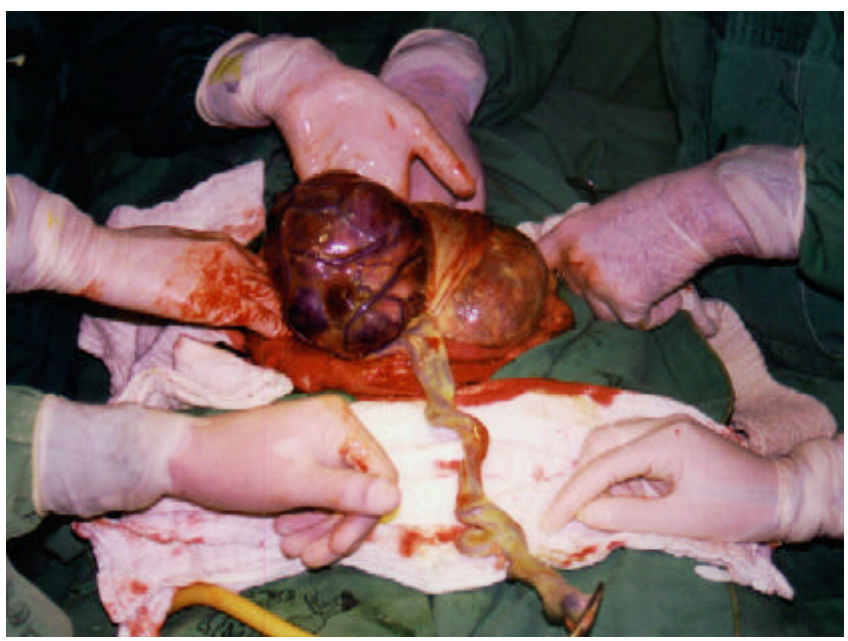

Figura 2 - Retirada completa da placenta, bastante volumosa.

A paciente apresentou evolução pósoperatória satisfatória, mantendo balanço hidroeletrolítico normal. A infusão de líquidos foi monitorizada por pressão venosa central (PVC) por meio de cateter na veia jugular direita. Exames laboratoriais (função renal, ionograma e gasometria) dentro da normalidade. Hemoglobina no $1^{\circ}$ dia pós-operatório (DPO) de $10 \mathrm{~g} \%$ e hematócrito de $30,9 \%$. O cateter epidural foi mantido nas primeiras 48 horas de pós-operatório, recebendo morfina e bupivacaína com 24 horas, em razão da queixa de dor. Presentes os ruídos hidroaéreos, iniciou alimentação oral com 12 horas de pós-operatório, inicialmente líquida e a seguir livre. Recebeu antibioticoterapia, $1 \mathrm{~g}$ de cefalotina a cada 6 horas por 24 horas e a seguir cefalexina na dosagem de $500 \mathrm{mg}$ a cada 6 horas por 7 dias. Administrou-se albendazol em virtude do diagnóstico de Strongiloides stercoralis no exame parasitológico de fezes. Obteve alta no 10 $\mathrm{DPO}$, em boas condições.

O recém-nascido, do sexo feminino, pesou 2.570 gramas e apresentou escores de Apgar de 3 no primeiro minuto, reagindo porém às manobras de reanimação. Os escores de Apgar no quinto e décimo minutos foram de respectivamente 6 e 8 . Não havia malformações ou deformidades aparentes. A idade gestacional estimada pelo método de Capurro foi de 37 semanas. Evoluiu com infecção precoce (perinatal), atribuída à amniorrexe prematura, recebendo anti-bioticoterapia por 10 dias (penicilina cristalina e gentamicina). Recebeu alta no 10 ㅇia de vida, em condições satisfatórias, com aleitamento materno exclusivo.

\section{Discussão}

Observou-se no presente caso evolução favorável da gestação abdominal, sem maiores dificuldades de extração fetal e retirada da placenta durante a laparotomia e com sobrevivência da mãe e do recém-nascido. Ressalte-se porém que esse tipo de resultado constitui a exceção, e não a regra, na história natural da gestação abdominal, em que advêm, em geral, complicações temiveis.

Enquanto na maioria dos casos a morte fetal é quase sempre infalivel ${ }^{2,5,11}$, a própria vida materna pode estar ameaçada, como sói ocorrer nos casos de hemorragia, decorrente quer do descolamento prematuro e espontâneo da placenta ${ }^{4}$, quer das manobras obstétricas para sua extração no ato operatório. A mortalidade materna descrita na literatura varia entre $5-20 \%{ }^{1,5,8}$. No estudo de Atrash et al. ${ }^{6}$, o risco de morte materna na presença de gestação abdominal foi 7,7 vezes maior que na gestação tubária e 90 vezes maior que na gestação uterina tópica.

Além da hemorragia, que sobressai como causa príncipe da morbimortalidade materna ${ }^{1,2}$, sobrevêm outras complicações no curso da gestação abdominal, como a infecção e, em menor freqüência, a anemia, hipofibrinogenemia, a obstrução intestinal, a embolia amniótica e até mesmo a formação de fístula âmniointestinal ${ }^{1,2,15,16}$. No caso ora relatado, encontramos associação com anemia e desnutrição materna, 
porém esses achados não resultaram, felizmente, em complicações maiores para o binômio mãe-feto.

Acreditamos que os bons resultados neste caso devam-se, outrossim, ao fato de que a placenta, ao se inserir quase completamente no omento, poupou as estruturas nobres, tendo sua extração sido sobremaneira facilitada no ato operatório. Não houve hemorragia importante e a própria anemia apresentada pela paciente sugeria um quadro crônico, associado também à desnutrição protéico-calórica. Além disso, a cirurgia foi realizada somente após equilíbrio das condições hemodinâmicas da paciente, efetuandose hemotransfusão e mantendo-se estocadas várias unidades de concentrado de hemácias. Esse preparo pré-operatório contribuiu sem dúvida para que a cirurgia se realizasse em condições satisfatórias.

Cumpre salientar, todavia, que o aspecto mais inusitado na evolução do presente caso foi o resultado neonatal, com extração de recémnascido vivo, a termo, sem malformações ou deformidades, e que logrou sobreviver não obstante a intercorrência de infecção perinatal, requerendo a administração de antibióticos.

$\mathrm{Na}$ verdade, são raríssimos os relatos na literatura de sobrevivência do concepto ${ }^{10,11,12,13,14}$, uma vez que o óbito fetal é extremamente comum $^{1,2,8,17}$, decorrente quer da insuficiência quer do descolamento da placenta. Reiteradas vezes encontra-se retardo do crescimento fetal e habitualmente os conceptos apresentam, ao nascimento características típicas do pós-datismo, como pele apergaminhada, ossos e unhas longos e aspecto de envelhecimento ${ }^{11}$.

Deformidades dos membros inferiores e da cabeça fetal também podem ser verificadas, devido ao contato permanente com estruturas sólidas maternas, sem a proteção conferida pelas paredes uterinas e pelo líquido amniótico ${ }^{2,7}$, pois o oligoâmnio representa achado freqüente. Nos eventuais casos em que se obtêm nascidos vivos, a mortalidade neonatal é muito elevada, quer por conta da prematuridade, quer da hipoplasia pulmonar ou da hipoxia ${ }^{1,2,8}$.

Vale ainda ressaltar outro aspecto incomum do caso que apresentamos, que é seu diagnóstico tardio, apesar de a paciente referir três consultas pré-natais em outro serviço e ter exame ultrasonográfico realizado por volta do $7^{\circ}$ mês. Embora Martin Jr e McCaul refiram que apenas 50\% dos casos têm diagnóstico pré-natal ${ }^{16}$, há que se considerar que o diagnóstico precoce continua sendo o ponto chave para evitar as deletérias conseqüências da gestação abdominal.

Deve-se lembrar que importantes alterações clínicas cursam com o evolver da gestação extra- uterina, em especial a dor abdominal inexplicada ${ }^{1,5,8}$, que era referida pela paciente como constante por toda a gravidez. Outros sintomas associados são as alterações gastrointestinais (náuseas, vômitos, constipação) e a presença de dor à movimentação fetal, que é em geral percebida nas partes altas do abdome $e^{1,10,11}$.

Ao exame físico, chama a atenção a superficialidade das partes fetais bem como a dor à palpação e à movimentação fetal. Não se percebem contorno nem contrações do útero, podendo-se ao toque combinado perceber, algumas vezes, o útero vazio ${ }^{10,11,16}$

A ultra-sonografia geralmente fecha o diagnóstico, em especial quando realizada em fases precoces da gestação, pois a termo o diagnóstico pode ser difícil mesmo para ultrasonografistas experientes. Os principais achados ecográficos característicos de gestação abdominal são a presença do feto e da placenta em localização extra-uterina, partes fetais muito próximas da parede abdominal e não-visualização do miométrio entre o feto e/ou a placenta e a bexiga materna ${ }^{18}$. São também freqüentes o oligoâmnio e as apresentações anômalas, encontrando-se habitualmente dificuldade na visibilização da placenta $^{18,19}$. Embora esses achados tenham sido referidos na ultra-sonografia realizada em nosso serviço, não foram descritos e, provavelmente, passaram despercebidos no exame anterior.

Em decorrência de todas essas peculiaridades, julgamos relevante a publicação do caso em tela, que foge em diversos pontos da evolução habitual das gravidezes abdominais avançadas, que per se já representam um evento bastante raro. Salientamos outrossim o êxito obtido em sua evolução, tanto do ponto de vista materno como neonatal.

\section{SUMMARY}

Term abdominal pregnancy with live fetus is an obstetrical rarity with high fetal and maternal morbidity and mortality. The authors present a case of abdominal pregnancy in a 43year-old woman. The diagnosis was made only at term (37 weeks) by clinical findings and echography. Exploratory laparotomy was performed and a living female newborn weighing 2,570 $\mathrm{g}$ was extracted. Apgar scores were 3, 6 and 8 at the 1st, 5th and 10th minutes, respectively. Placenta was inserted in the omentum and was removed without complications. Postoperative course was uneventful and both mother and child were discharged healthy.

KEY WORDS: Ectopic pregnancy. Abdominal Pregnancy. Pregnancy complications. Fetal growth restriction. 


\section{Referências}

1. Rahman MS, Al Suleiman AS, Rahman J, Al Sibai $\mathrm{MH}$. Advanced abdominal pregnancy observations in 10 cases. Obstet Gynecol 1982; 59:366-72.

2. White RG. Advanced abdominal pregnancy - a review of 23 cases. Ir J Med Sci 1989; 158:77-8.

3. Alto W. Is there a greater incidence of abdominal pregnancy in developing countries? Report of four cases. Med J Aust 1989; 151:412,412-14.

4. Hallat JG, Grove JA. Abdominal pregnancy: a study of twenty-one consecutive cases. Am J Obstet Gynecol 1985; 152: 444-9.

5. Mbura JS, Mgaya HN. Advanced abdominal pregnancy in Muhimbili Medical Centre, Tanzania. Int $\mathrm{J}$ Gynecol Obstet 1986; 24:169-76.

6. Atrash HK, Friede A, Hougue CJR. Abdominal pregnancy in the United States: frequency and maternal mortality. Obstet Gynecol 1987; 69:333-7.

7. Cartwright PS, Brown JE, Davis RJ, Thieme GA, Boehm FH. Advanced abdominal pregnancy associated with fetal pulmonary hypoplasia: report of a case. Am J Obstet Gynecol 1986; 155:396-7.

8. Golan A, Sandbank O, Andronikou A, Rubin A. Advanced extra-uterine pregnancy. Acta Obstet Gynecol Scand 1985; 64: 21-5.

9. Foster HW, Moore DT. Abdominal pregnancy. Report of 12 cases. Obstet Gynecol 1967; 30:249-52.
10.Costa CFF, Leite A, Alencar AV, Lomachinsky G, Maia VOA. Gravidez abdominal com feto vivo. Rev Bras Ginecol Obstet 1985; 7:49-52.

11. Costa CFF. Diagnóstico e tratamento da prenhez ectópica abdominal tardia. Femina 1986; 14:797-800.

12.Costa CFF, Leite E, Welkovic S. Gravidez abdominal com feto vivo de 36 semanas. Rev Bras Ginecol Obstet 1993; 15:64-6.

13.Alencar Jr CA, Carvalho Neto, RH, Alencar, MF, Seabra M CM, Arrais RBM, Almeida FML, et al. Gravidez abdominal avançada com feto vivo: diagnóstico ecográfico e evolução perinatal. Rev Bras Ginecol Obstet 1994;16:43-8.

14. Mendonça D, Mendonça F, Ramos AA, Ney R, Paiva CSM, Guedes Filho A, et al. Conduta inusitada na hemorragia severa por rotura de gravidez abdominal. J Bras Ginecol 1993;102:85-7.

15.Brown RC, Varner MW. Anemia as a sign of abdominal pregnancy. Am Fam Physician 1984; 29:135-8.

16. Martin JN Jr, McCaul JF $4^{\text {th }}$. Emergent management of abdominal pregnancy. Clin Obstet Gynecol 1990; 33: 438-47.

17.Delke I, Veridiano NP, Tancer ML. Abdominal pregnancy: review of current management and addition of 10 cases. Obstet Gynecol 1982; 60: 200-4.

18.Stanley JH, Horger EO $3^{\mathrm{d}}$, Fagan CJ, Andriole JG, Fleischer AC. Sonographic findings in abdominal pregnancy. AJR (Am J Roentgenol) 1986; $147: 1043-6$

19. Martin JN Jr, Sessuns JK, Martin RW, Pryor JA, Morrison JC. Abdominal pregnancy: current concepts of management. Obstet Gynecol 1988;71:549-57. 